\title{
The closed model of the universe with relativistic redshift
}

\author{
Zahid Zakir $^{1}$
}

\begin{abstract}
Starting from the relativistic dynamics of homogeneous expanding ball, a relativistic evolution equation for the closed model of the Universe is formulated which correctly takes into account a relativistic time dilation of proper times due to different expansion speeds at different epochs. The hypersurface of simultaneity of the Universe is spherically-symmetric only in the rest frame of 3sphere's centre and, consequently, the evolution is described in world time t of this frame of reference. This relativistic evolution equation coincided with Einstein's equation for a 3-sphere expanding in 4space (without dark energy) and has a simple exact solution. There is a relativistic redshift leading to new relation of redshift with the scale factor which then gives new "luminosity-redshift" relation well describing the data for Type 1a supernovae at admissible parameters. By using the initial data - present radius and expansion speed - other properties and evolution of the Universe are predicted. The model predicts that the observing baryon density is one third of total matter density and remaining two third is dark matter density. The accounting of stasis effects slightly changes the parameters only.

PACS: 04.20.Cv, 98.80.-k, 98.80.Jk 95.30.Sf, 97.60.Lf, 98.35.Jk, 98.54.-h, 98.80.-k, 04.60.-m

Key words: cosmological models, redshift, supernovae, galaxy clusters, dark matter, dark energy
\end{abstract}

\section{Content}

Introduction .24

1. Relativistic dynamics of a ball and new closed model .......................................................25

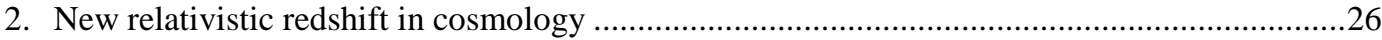

3. Structure and parameters of the universe in the new model ..................................................29

4. The stasis effects for redshift and intensity at crossing of clusters ..........................................31

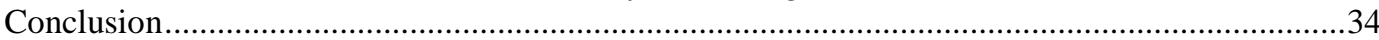

Appendix. The Friedmann model from non-relativistic dynamics of a ball ...............................35

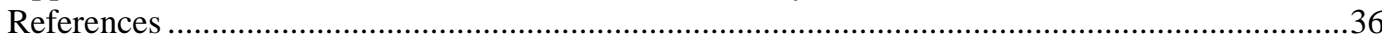

\section{Introduction}

In relativistic cosmology [1-5], based on the cosmological principle and energy conservation for matter, the Big Bang - an initial dense and hot state of the Universe - is directly compatible by a closed model only. Flat and open models, supposing actually infinite space, in fact, lead to direct or hidden contradictions and require new hypotheses and boundary conditions.

Last decades the data on redshifts $z$ and luminosities of Type la supernovae, taken as a «standard candle», have shown that objects with increasing $z$ become dimmer, than follows from the standard Friedmann models [6,7]. The "explanationd" of this dimming by hypotheses about flatness and a dark energy accelerating the expansion, also are based on the Friedmann models and introduce to them unknown form of energy only.

\footnotetext{
${ }^{1}$ Centre for Theoretical Physics and Astrophyics, Tashkent, Uzbekistan; zahidzakir@theor-phys.org
} 
However, already the stasis effects for frequency and luminosity of photons at crossing the galaxy clusters [8] show that there may be more complicated relation of $z$ with the scale factor due to unaccounted in the model relativistic effects.

In other side, incompleteness of the Friedmann models shows also the fact that the evolution equation in them follows from the nonrelativistic dynamics of a ball of enough small radius, extrapolated to entire Universe [5] (see Appendix). But for a ball of enough large radius the expansion velocity of its surface becomes so high that relativistic effects should essentially modify the evolution equation. For this reason, only a relativistic evolution equation of the ball can be extrapolated to the whole Universe. The aims of the present paper are finding such equation and studying its consequences for cosmology.

In the paper it is shown that such consecutive version of the closed model (without dark energy), more accurately accounting the relativistic effects and 3-sphere's geometry, leads to new "luminosity-redshift" relation well describing the observations. The model predicts new values for total matter density, also coinciding with observations.

In Sections 1-3 the basic relations of the new model are presented and comparison with observations is performed, in Section 4 the stasis effects corrections are considered. In Appendix main relations of the former closed model are presented too.

\section{Relativistic dynamics of a ball and new closed model}

The standard version of the closed model, shortly described in Appendix, follows from the nonrelativistic dynamics of an expanding ball [5] based on a set of approximations. At description of ball's evolution without restriction of its size, and especially for the whole Universe, the approximation of weak gravitation remains valid, but the nonrelativistic approximation becomes already inadmissible.

Formally the expanding 3-sphere differs from an expanding 2-sphere only by replacing of an angular linear element on 2-sphere $d \Omega_{(2)}^{2}$ by an angular linear element on 3-sphere $d \Omega_{(3)}^{2}$. The radial evolution, mainly interesting for us, should be almost similar in both cases and thus, at first, we will consider more clear case of 2-sphere. If one turns to one of comoving to 2-sphere local-inertial frames, all such local frames in other points of the sphere will move w.r.t. it with different velocities. Thus, due to relativistic contraction, in the comoving to this point inertial frame 2-sphere transforms to ellipsoid. Therefore, in fact 2-sphere is spherically-symmetric only in the rest frame of it's centre, where a linear element on its surface, expanding with velocity $v=d r / d t$, takes the form:

$$
d s^{2}=d t^{2}-d r^{2}-r^{2}(t) \cdot d \Omega_{(2)}^{2}=d t^{2}\left(1-v^{2}\right)-r^{2}(t) \cdot d \Omega_{(2)}^{2} .
$$

Here $d \tau=d t \sqrt{1-v^{2}}$ is the proper time interval on 2-sphere.

At large distances on 2-sphere with $r(t) \theta$, as well as on 3-sphere with $a(t) \chi$, it is necessary to consider the relativistic dilation of proper times due to high expansion velocity and difference of this velocity at different times.

For this purpose it is necessary turn to relativistic kinematics with different intervals of proper time $d \tau(a)$ at different times and to introduce world time $t$ of a rest frame of 3-sphere's "centre". At the maximal expansion of the ball at $r=r_{m}$, when all particles will rested w.r.t. each other, as well as the Universe as whole, it will possible unambiguous synchronization of clocks in the whole Universe.

Proper time $t$ of this frame $K_{m}$, where the symbolic "centre" of the 3 -sphere is rested, is natural world time of the closed Universe. Moreover, only in this frame the 
Universe looks like as a 3-sphere. With the account of these facts, further we will describe the expansion of the Universe in $K_{m}$, where world time $t$ is measured at any epoch by the comoving to 3 -sphere and synchronously going coordinate clocks.

Thus, proper time on the 3 -sphere we can express in terms of global world time as in (1) (further $\dot{a} \equiv d a / d t$ ):

$$
d \tau=d t \sqrt{1-\dot{a}^{2}}
$$

Then, the linear element on 3 -sphere becomes similar to (1):

$$
d s^{2}=d t^{2}-d a^{2}-a^{2}(t) \cdot d \Omega_{(3)}^{2}=d t^{2}\left(1-\dot{a}^{2}\right)-a^{2}(t) \cdot d \Omega_{(3)}^{2} .
$$

By substituting (2) into (55), we come to the evolution equation for the Universe:

$$
\frac{\dot{a}^{2}}{1-\dot{a}^{2}}=\frac{a_{m}}{a}-1
$$

An evolution equation similar to (4) has been obtained earlier by C.-G. Park (the equation (35b) in [9]) from Einstein's equation with the linear element (3) and studied by using Eq. (59) without considered below relativistic redshift.

\section{New relativistic redshift in cosmology}

In the present paper consequences of the relativistic dilation of proper time due to expansion velocity of 3-sphere and difference of this velocity at different epochs will be considered more consecutively. This time dilation, taking into account (2) and (4), is given by the expressions:

$$
d \tau(a)=d t \sqrt{\frac{a}{a_{m}}}, \quad \frac{\delta \tau\left(a_{0}\right)}{\delta \tau\left(a_{z}\right)}=\frac{d t_{0}}{d t_{z}} \sqrt{\frac{a_{0}}{a_{z}}} .
$$

From (5) and the equations for photon's trajectory:

$$
d s^{2}=d t^{2}\left(1-\dot{a}^{2}\right)-a^{2}(t) \cdot d \chi^{2}=0
$$

there follow the same formulas as in Eqs. (57)-(58).

Redshift $z$ also is defined as before through the ratio of wavelengths (59), however, now its relation with the scale factor modifies and from (5) we obtain the new relation:

$$
1+z=\frac{\lambda_{r}}{\lambda_{e}}=\frac{\delta t_{r}}{\delta t_{e}} \sqrt{\frac{a_{0}}{a_{z}}} .
$$

At emitting and receiving of radiation in a resting 3-sphere it would be $\delta t_{r}=\delta t_{e}$. But, since in the expanding 3-sphere distance between two points is stretching and thus a world time period of wave, i.e. an interval between wave crests, also is stretching proportional to the scale factor:

$$
\frac{\delta t_{r}}{\delta t_{e}}=\frac{a_{0}}{a_{z}}
$$

Notice, that now $\delta \chi_{r} \neq \delta \chi_{e}$ due to the relativistic stretching of wavelengths.

From Eqs. (7)-(8) we obtain new formulas relating redshift with scale factor:

$$
1+z=\frac{a_{0}^{3 / 2}}{a_{z}^{3 / 2}}, \quad \frac{a_{0}}{a_{z}}=(1+z)^{2 / 3} .
$$

Thus, at given $a$ the relativistic dilation of proper times (or the transverse Doppler effect) at earlier epochs leads to increasing of redshift of photons to $\left(a_{0} / a_{z}\right)^{1 / 2}$ or $(1+z)^{1 / 3}$. 
Apparent luminosity $l$, by definition, is related by a solid angle by which the source is visible. At propagation of beam of photons in 3-sphere expanding with radial velocity $\dot{a}$, orthogonal to the 3 -sphere, there it takes place relativistic aberration. In the relativistic kinematics an element of solid angle $d \varphi d(\cos \theta)$ in a rest fram of the source differs on the same solid angle in the rest frame of an observer $d \varphi d\left(\cos \theta^{\prime}\right)$ due to its transformation as:

$$
d\left(\cos \theta^{\prime}\right)=\frac{1-v^{2}}{(1-v \cos \theta)^{2}} d(\cos \theta)
$$

At emitting of photons (in transverse direction to velocity) on 2-sphere at radius $r_{1}$ and velocity $v\left(r_{1}\right)$, and receiving at radius $r_{2}$ and velocity $v\left(r_{2}\right)$, due to such difference of solid angles, the ratio of apparent luminosities will contain the relativistic factor:

$$
\frac{d \Omega_{e}}{d \Omega_{r}}=\frac{1-v_{1}^{2}}{1-v_{2}^{2}} .
$$

If $v\left(r_{2}\right)<v\left(r_{1}\right)$, we have $d\left(\cos \theta_{2}\right)>d\left(\cos \theta_{2}\right)$ and consequently $l_{2}<l_{1}$.

Similarly, on 3 -sphere the ratio of solid angle elements at the receiver $d \Omega_{r}$ and at the source $d \Omega_{e}$ will differ to the relativistic aberration factor:

$$
\frac{d \Omega_{e}}{d \Omega_{r}}=\frac{1-\dot{a}_{z}^{2}}{1-\dot{a}_{0}^{2}}=\frac{a_{z}}{a_{0}}=\frac{1}{(1+z)^{2 / 3}} .
$$

Thus, the relativistic aberration reduces apparent luminosity $l$, which is expressed by multiplication to the additional factor $(1+z)^{-2 / 3}$.

Taking into account Eq. (12), instead of Eq. (61), we obtain:

$$
l=\frac{L}{4 \pi a_{0}^{2} \sin ^{2} \chi_{z}} \cdot \frac{1}{(1+z)^{8 / 3}} .
$$

The luminosity distance $d_{p, 0}$, thus, is equal to:

$$
d_{p, 0}=a_{0} \sin \chi_{z} \cdot(1+z)^{4 / 3}=10^{-5+(m-M) / 5} \mathrm{Mpc},
$$

from which we come to the expression for the distance modulus:

$$
\mu=5 \lg \left[a_{0}(1+z)^{4 / 3} \cdot \sin \chi_{z}\right]+25 \text {. }
$$

By substituting (58) and (9) into (15), we obtain new formula for the distance modulus:

$$
\mu=5 \lg \{3(1-b) Z[Z-1-(1-2 b)(\sqrt{(Z-b) /(1-b)}-1)]\}+A,
$$

where $\mathrm{Z} \equiv(1+z)^{2 / 3}$ and

$$
A=5 \lg \left(c / H_{o b s}\right)+25, \quad H_{o b s}=\frac{3}{2} H_{0}=\frac{3 c}{2 a_{0}} \frac{1}{\sqrt{b^{-1}-1}} .
$$

This formula (16) replaces Mattig's formula (64) obtained without accounting the relativistic effects. Further we will use $h_{0}$ introduced as $H_{o b s}=h_{0} \cdot 100 \mathrm{~km} \cdot \mathrm{sec}^{-1} \mathrm{Mpc}^{-1}$.

At $b=1 / 2$ Eq. (16) simplifies:

$$
\mu=5 \lg [1.5 Z(Z-1)]+A \text {. }
$$

At small $z$ a series of Eq. (16) gives:

$$
\mu \simeq 5 \lg \left(z+\gamma z^{2}\right)+A
$$




$$
\gamma(b)=(4-5 b) / 6(1-b), \quad \gamma(1 / 2)=1 / 2
$$

For comparison with observations of the new $\mu-z$ relation (16) we will use the data set for 601 Type 1a supernovae (SN 1a): 580 objects from Union 2.1 compilation [10] (for 15 objects of which we use the data from [14]), 3 new objects with $z_{s}=1.55$ [11], $z_{s}=1.71$ [12], $z_{s}=1.91$ [13], and 33 objects from the "pure" set [15].

The constant $h_{0}$ in (16) is model-independent parameter which in any model should be fixed at small $z$ in the interval $0.03<z<0.1$, where local velocities already do not sufficiently disturb the data and non-linear on $z$ contributions are still small. In this interval 95 objects give the value $h_{0}=0.698$ by good precision $\left(\chi^{2} / N \simeq 0.87\right.$ ).

At known $h_{0}$ the model allows us to express present proper age of the Universe $\tau_{0}$ through $b$ (see the formula (24)). Thus, we can fix the parameter $b$ from the observational limits to $\tau_{0}$. The period of time when the dust matter approximation was valid is not less than $13.2<\tau_{0}<13.7 \mathrm{Gyr}$, which gives us $0.25>b>0.11$.

As shows Fig. 1 (red line), at the values of parameters:

$$
h_{0}=0.698, \quad b=0.18 \pm 0.07
$$

the model predicts for the data at $z>0.1$ the values coinciding with the observations very well up to $z \sim 1.9$ with $\chi^{2} / N \simeq 1.03$.

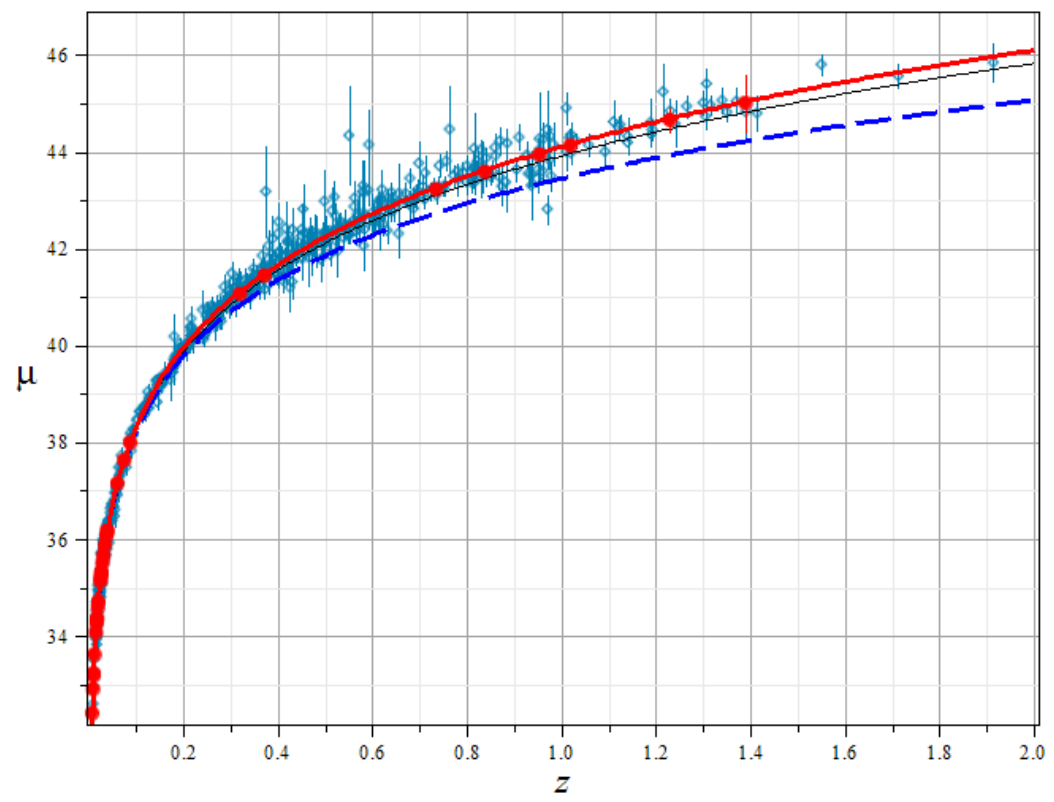

Puc.1. Dependence of the distance modulus $\mu$ on redshift $z$. Points - are observational data for 568 SN 1a from [10-13] (dark blue rhombs) and 33 "pure" SN 1a from [14] (red circles). Red curve - according Eq. (16) at $h_{0}=0.698$, $b=0.20 \pm 0.10$, a thin curve - according to Eq. (18), and blue dashed line - from Mattig's formula (64) with $q_{0}=1 / 2(1-b)=0.63$.

Notice, that due to a changing of distances up to sources with redshift $z$ w.r.t. former models in flat space, the results of observations should be corrected with the account of additional absorption and scattering. 
Corrections of the stasis effects to $z$ and luminosity of radiation at passing of galaxy clusters due to switching out from the cosmological expansion flow [8] are even more essential and they will be considered in Section 4.

\section{Structure and parameters of the universe in the new model}

In the closed model basic properties of the Universe and its evolution are defined across two parameters: $a_{0}$ and $a_{m}$. Relations between the expansion velocity, radius and time follow from (4):

$$
\begin{gathered}
\dot{a}= \pm \sqrt{1-a / a_{m}}, \dot{a}_{0}=\sqrt{1-b}, \quad a=t-t^{2} / 4 a_{m}, \\
t=2 a_{m}\left(1-\sqrt{1-a / a_{m}}\right), \quad t_{0}=2 a_{m}(1-\sqrt{1-b}) .
\end{gathered}
$$

World time from the beginning up to the maximum of expansion is $t_{m}=2 a_{m}$ and, besides, at early epochs $\left(t \ll a_{m}\right)$ the expansion lasts under the linear law $a(t) \sim t$. Proper time in a comoving frame from the beginning of expansion is:

$$
\begin{gathered}
\tau=\int_{0}^{t} d t \sqrt{1-\dot{a}^{2}}=-\int_{0}^{a} \frac{d a \sqrt{a}}{\sqrt{a_{m}-a}}=a_{m} \arcsin \sqrt{\frac{a}{a_{m}}}-\sqrt{a\left(a_{m}-a\right)}, \\
\tau_{0}=a_{m}(\arcsin \sqrt{b}-\sqrt{b(1-b)}) .
\end{gathered}
$$

The proper time interval from Big Bang up to stopping at $a\left(\tau_{m}\right)=a_{m}$, thus, is equal to $\tau_{m}=a_{m} \pi / 2$ and it is less than corresponding world time: $\tau_{m}<t_{m}$. Though these expressions are obtained without accounting of energy density and pressure of radiation, nevertheless, they give the age $\tau_{0}$ close enough to the real age of the Universe, since a time when radiation dominated is sufficiently less of total age.

In any closed model it is interesting to determine: can a light signal have enough time to circumvent the spherical Universe during expansion? For an angle $\chi$ we obtain:

$$
\begin{gathered}
\chi_{0}=\int_{0}^{t_{0}} \frac{d t \sqrt{1-\dot{a}^{2}}}{a}=2 \int_{0}^{t_{0}} \frac{d t}{\sqrt{t\left(4 a_{m}-t\right)}}=2 \arccos \left(1-\frac{t_{0}}{2 a_{m}}\right) \\
\cos \frac{\chi_{0}}{2}=1-\frac{t_{0}}{2 a_{m}} .
\end{gathered}
$$

As we see, the photons emitted at the beginning of expansions to the time of maximal expansion ( $t_{m}=2 a_{m}, b_{m}=1$ ) have time to reach only from one pole up to opposite pole ( $\chi=\pi$ ). From Eq. (57) we also see that since while $b \simeq 0.18$, the observing relic radiation has been emitted from regions closer than equator of the 3 -sphere:

$$
\chi_{0}=\frac{\pi}{2}-\arcsin (1-2 b) \simeq 0.88 \simeq 50^{\circ} .
$$

Therefore the present version of the closed model does not predict direct effects of periodicity.

For the parameter $H_{z}$ we obtain the expressions in terms of world time $(c \neq 1)$ :

$$
H_{z}=\frac{\dot{a}_{z}}{a_{z}}=\frac{c}{a_{z}} \sqrt{1-\frac{a_{z}}{a_{m}}}, \quad H_{0}=\frac{c}{a_{0}} \sqrt{1-b}=\frac{2}{3} H_{o b s} \sqrt{b},
$$

and proper time of comoving to 3 -sphere observer: 


$$
\bar{H}_{z}=\frac{1}{a_{z}} \frac{d a_{z}}{d \tau}=\frac{H_{z}}{\sqrt{1-\dot{a}_{z}^{2}}}=H_{z} \sqrt{\frac{a_{m}}{a_{z}}}=H_{o b s} \frac{2(1+z)}{3 \sqrt{1-b}} \sqrt{1-\frac{b}{(1+z)^{2 / 3}}} .
$$

Further instead of $a_{0}$ and $a_{m}$ it is more convenient to use $b=a_{0} / a_{m}$ and $H_{o b s}$ from Eq. (16) for distance modulus and, thus, we have:

$$
a_{0}=\frac{3 c}{2 H_{o b s}} \sqrt{b^{-1}-1}, \quad a_{m}=\frac{a_{0}}{b} .
$$

In the closed model there is the restriction:

$$
b \equiv \frac{a_{0}}{a_{m}}=\frac{a_{0} c^{2}}{2 G M}=\frac{c^{2}}{G \rho_{0}\left(2 \pi a_{0}\right)^{2}}<1 .
$$

That gives lower limit for the total matter density $\rho_{0}$ :

$$
\rho_{0}>\rho_{3 c}=\frac{c^{2}}{G\left(2 \pi a_{0}\right)^{2}}=\frac{1}{9 \pi^{2} G} \cdot \frac{H_{o b s}^{2}}{\left(b^{-1}-1\right)}=\frac{8}{27 \pi\left(b^{-1}-1\right)} \rho_{c} .
$$

This new critical density $\rho_{3 c}$ jf the closed model is more than 40 times less than the critical density of Friedmann model $\rho_{c}=3 H_{o b s}^{2} / 8 \pi G \approx h^{2} \cdot 1.88 \cdot 10^{-29} \mathrm{~g} \cdot \mathrm{sm}^{-3}$.

Total matter density $\rho_{m}=\rho_{0}$ at present directly follows from (32) and its value is predicted by the model. At the accepted values of parameters of model it in $b^{-1}=2.3$ times more than new critical density $\rho_{3 c}$ and is equal to:

$$
\rho_{0}=\frac{c^{2}}{G b} \frac{1}{\left(2 \pi a_{0}\right)^{2}}=\frac{\rho_{3 c}}{b}
$$

Baryonic matter density $\rho_{b}$, following from the analysis the observetional data, is about $\rho_{b} \simeq 3.4 \times 10^{-31} \mathrm{~g} \cdot \mathrm{sm}^{-3}$ i.e. corresponds to about $30 \%$ of $\rho_{m}$.

The dark matter density $\rho_{d m}$ then is difference between $\rho_{m}$ and $\rho_{b}$, i.e. $\rho_{d m}=\rho_{m}-\rho_{b}$. Thus, its predicted by the model value is about $70 \%$ from $\rho_{0}$.

The total mass of the Universe $M$ is finite, conserved and defined by $a_{m}$ :

$$
M=a_{m} c^{2} / 2 G \sim 1.6 \cdot 10^{57} .
$$

Mean number of galaxies in the Universe is finite and can be estimated by dividing the fraction of galaxies about $0.03 M$ in $M$ to mean mass of a galaxy $\bar{M}_{\text {gal }} \sim 10^{11} M_{\mathrm{o}} \sim 10^{44} \mathrm{~g}$. Such estimation gives $N_{\text {gal }} \geq 0.03 \mathrm{M} / \bar{M}_{\text {gal }} \sim 2 \cdot 10^{11}$ which quite close to the observational estimations.

The parameters of the Universe at (21) and close them values of parameters of the model are presented in Table 1.

The model predicts also series of effects related by geometry of 3-sphere, kinematics with relativistic effects, and by combination of these circumstances. The geometrical effects of the spherical world reveal as universal properties of objects not depending from their nature. 


\begin{tabular}{|cc|ccc|c|c|c|c|c|c|c|c|}
\hline$h_{o}$ & $b$ & $a$ & $a_{\max }$ & $\tau$ & $\tau_{\max }$ & $t$ & $t_{\max }$ & $\rho_{m}$ & $\rho_{b} / \rho_{m}$ & $\rho_{d m} / \rho_{m}$ & $M$ \\
\hline 0,670 & 0,15 & 51 & 342 & 13,9 & 537 & 53 & 685 & 10 & $36 \%$ & $64 \%$ & 22 \\
0,680 & 0,20 & 43 & 216 & 13,7 & 339 & 46 & 431 & 10 & $33 \%$ & $67 \%$ & 14 \\
0,680 & 0,25 & 37 & 149 & 13,5 & 235 & 40 & 299 & 11 & $31 \%$ & $69 \%$ & 10 \\
\hline 0,698 & 0,11 & 60 & 543 & 13,7 & 853 & 62 & 1087 & 10 & $35 \%$ & $65 \%$ & 35 \\
0,698 & 0,18 & 45 & 249 & 13,4 & 391 & 47 & 498 & 10 & $33 \%$ & $67 \%$ & 16 \\
0,698 & 0,25 & 36 & 146 & 13,2 & 229 & 39 & 291 & 11 & $30 \%$ & $70 \%$ & 9 \\
\hline 0,710 & 0,15 & 49 & 328 & 13,3 & 515 & 51 & 656 & 10 & $33 \%$ & $67 \%$ & 21 \\
0,710 & 0,20 & 41 & 207 & 13,1 & 324 & 44 & 413 & 11 & $31 \%$ & $69 \%$ & 13 \\
0,710 & 0,25 & 36 & 143 & 13,0 & 225 & 38 & 286 & 12 & $29 \%$ & $71 \%$ & 9 \\
\hline
\end{tabular}

Table 1. Parameters of the Universe in closed model with relativistic redshift (without stasis effects corrections). Radii $a, a_{\max }$ in Gly, ages $\tau, \tau_{\max }, t, t_{\max }$ in Gy, total matter density $\rho_{m}$ in $10^{-31} \mathrm{~g} \cdot \mathrm{sm}^{-3}$, total mass $M$ in $10^{56} \mathrm{~g}$.

\section{The stasis effects for redshift and intensity at crossing of clusters}

In the papers [8] new cosmological effects were studied related by the frequency and luminosity stasis of radiation due to switching out from the expansion flow at crossing the gravitationally-bound regions (GBR), largest of which are galaxy clusters. At multiple crossing the clusters these effects lead to sufficient decreasing of observable (spectroscopic) redshifts $z_{s}$ and magnification of apparent luminosity of sources. A normal redshift $z$, directly related to the scale factor, have photons uncrossed the clusters and they give true distance up to the sources, larger than those which follow from $z_{s}$. The effects increase with distance due to growng number of clusters crossed by radiation and smaller intercluster distances at earlier epochs.

Radius of GBR $r_{s}$ is determined by a point of "zero acceleration":

$$
H_{0}^{2} r_{s}=\frac{G M}{r_{s}^{2}}, \quad r_{s}=\frac{(G M)^{1 / 3}}{H_{0}^{2 / 3}} .
$$

Supposing that varying of distances between clusters are mainly due to the cosmological expansion, we obtain:

$$
\Delta l_{c}=\Delta l_{0 c} \frac{a}{a_{0}}, \quad \Delta l_{g}=\Delta l_{g 0} \frac{H_{0}^{2 / 3}}{H^{2 / 3}}, f=\frac{\Delta l_{g}}{\Delta l_{c}}=f_{0} \frac{a_{0} H_{0}^{2 / 3}}{a H^{2 / 3}} .
$$

The apparent (spectroscopic) redshift $z_{s}$ is defined by the ratio of proper wavelengths of photons at receiving $\left(\lambda_{r}\right)$ and emitting $\left(\lambda_{e}\right)$, whereas the ratio $a_{0}$ to $a_{z}$ defines a normal ("effective") redshift $z$ which photons would have if they did not crossed GBR. From (9) we have:

$$
\frac{\lambda_{r}}{\lambda_{e}}=1+z_{s}, \quad \frac{\bar{\lambda}_{r}}{\lambda_{e}}=1+z
$$

In a homogeneous world these two definitions coincide, however in the presence of GBRs these definitions are inequivalent and $z_{s}<z$.

At crossing by photons the intercluster distance $\Delta l_{c}$ the relativistic redshift by the factor $\left(a_{0} / a_{z}\right)^{1 / 2}$ happens everywhere, whereas the stretching of wavelength $\lambda$ due to 
expansion happens only out of $\Delta l_{g}$, i.e. in part of this distance equal to $(1-f) \Delta l_{c}$. As the result, it takes place the equation:

$$
\frac{d \lambda}{\lambda}=\frac{3}{2} \frac{d a}{a}-f \frac{d a}{a} .
$$

Using (37), this equation we rewrite in the form:

$$
\frac{d \lambda}{\lambda}=\frac{3}{2} \frac{d a}{a}-\bar{w} \frac{d a}{a^{2} H^{2 / 3}},
$$

where $\bar{w}=f_{0} a_{0} H_{0}^{2 / 3}$. In the new version of the closed model we have:

$$
H=\frac{\dot{a}}{a \sqrt{1-\dot{a}^{2}}}=\frac{a_{m}^{1 / 2} c}{a^{3 / 2}}\left(1-a / a_{m}\right)^{1 / 2}, H_{0}=\frac{c}{a_{0}}\left(b^{-1}-1\right)^{1 / 2}
$$

and the equation (40) turns to:

$$
\frac{d \lambda}{\lambda}=\frac{3}{2} \frac{d a}{a}-w \frac{d a}{a\left(1-a / a_{m}\right)^{1 / 3}},
$$

where $w=f_{0} y_{0}, y_{0}=(1-b)^{1 / 3}$ and $b=a_{0} / a_{m}$. Integration of this equation gives the required relation between $z_{s}$ and $z$ :

$$
1+z_{s}=(1+z) \cdot \frac{\exp \left\{3^{1 / 2} w \cdot \arctan \left[\left(1+2 y_{z}\right) / 3^{1 / 2}\right]\right\}}{G \cdot\left[1+3 y_{z} /\left(1-y_{z}\right)^{2}\right]^{w / 2}},
$$

where:

$$
\begin{aligned}
& y=\left(1-a / a_{m}\right)^{1 / 3}=\left[1-b /(1+z)^{2 / 3}\right]^{1 / 3}, \\
& G=\frac{\exp \left\{3^{1 / 2} w \cdot \arctan \left[\left(1+2 y_{0}\right) / 3^{1 / 2}\right]\right\}}{\left[1+3 y_{0} /\left(1-y_{0}\right)^{2}\right]^{w / 2}} .
\end{aligned}
$$

For expression $z$ through $z_{s}$ it is necessary to invert (43) and we will do that by approximation. The dependence $z_{s}(z)$ is smooth, so by taking first two terms of the series (43), and by introducing a correction factor $\alpha$, we obtain:

$$
z_{s} \simeq K z+Q z^{2}
$$

where:

$$
K=1-\frac{2}{3} f_{0}, \quad Q=-\frac{\alpha f_{0}}{27}\left(11-\frac{2}{1-b}+6 f_{0}\right) .
$$

Further for each combination of parameters $b, f_{0}$ in (45) we select such $\alpha$, which leads to a curve close to (43) at $z_{s}<2$ and then from (45) obtain the relation:

$$
z \simeq \frac{K}{2 Q}\left(\sqrt{1+\frac{4 Q \cdot z_{s}}{K^{2}}}-1\right)
$$

The stasis effects in GBR change apparent luminosity too. To radiation from a source of absolute luminosity $L$ and uncrossed through GBRs there would correspond apparent luminosity $\bar{l}=L /\left[4 \pi d_{0}^{2} \cdot(1+z)^{2}\right]$. If the flow crossed through GBRs, it is necessary to express $\bar{l}$ through apparent luminosity $l$ [8].

The magnification of $l$ due to a smaller stretching of an arrival time of photons and smaller redshift of their energy is given by the coefficient $B_{\delta z}^{2}$ : 


$$
B_{\delta z}^{2}=\left(\frac{a_{0} / a_{z}}{\lambda_{z} / \lambda_{0}}\right)^{2}=\left(\frac{1+z}{1+z_{s}}\right)^{2} .
$$

The normal beam outside the cluster with a solid angle $\pi \varepsilon^{2}$ will expands more than interior one and there will be a waist of the beam due to the stasis effect, so the corresponding magnification of $l$ expresses the coefficient $C_{\delta \varepsilon}$ :

$$
C_{\delta \varepsilon}=\frac{\pi \varepsilon_{z}^{2}}{\pi \varepsilon_{\bar{z}}^{2}}=\left(\frac{1+z}{1+z_{s}}\right)^{4 / 3}
$$

By introducing also the coefficient $D_{\delta z}$ of decreasing of apparent luminosity at additional absorption and scattering due to larger distance to sources, for the true luminosity of the source $\bar{l}$ we obtain:

$$
\bar{l}=l \cdot \frac{D_{\delta z}}{B_{\delta z}^{2} C_{\delta \varepsilon}^{2}}=l \cdot\left(\frac{1+z_{s}}{1+z}\right)^{10 / 3} D_{\delta z},
$$

Notice, that at $z \ll 1$ we have $z_{s} \sim\left(1-2 f_{0} / 3\right) \cdot z+O\left(\bar{z}^{2}\right)$, which leads to the renormalization of $H_{0}$ or absolute magnitude $M$ :

$$
\bar{H}_{0}=\frac{H_{o b s}}{1-2 f_{0} / 3} \text { or } \bar{M}=M+5 \lg \left(1-2 f_{0} / 3\right) \text {. }
$$

Corrections to absorption and radiation scattering at crossing of galos of galaxies usually are taken nearby $\Delta m \approx 0.03$ at $z_{s}=1.0$. By using this correction, for true (undistorted by clusters) distance modulus we obtain the expression [8]:

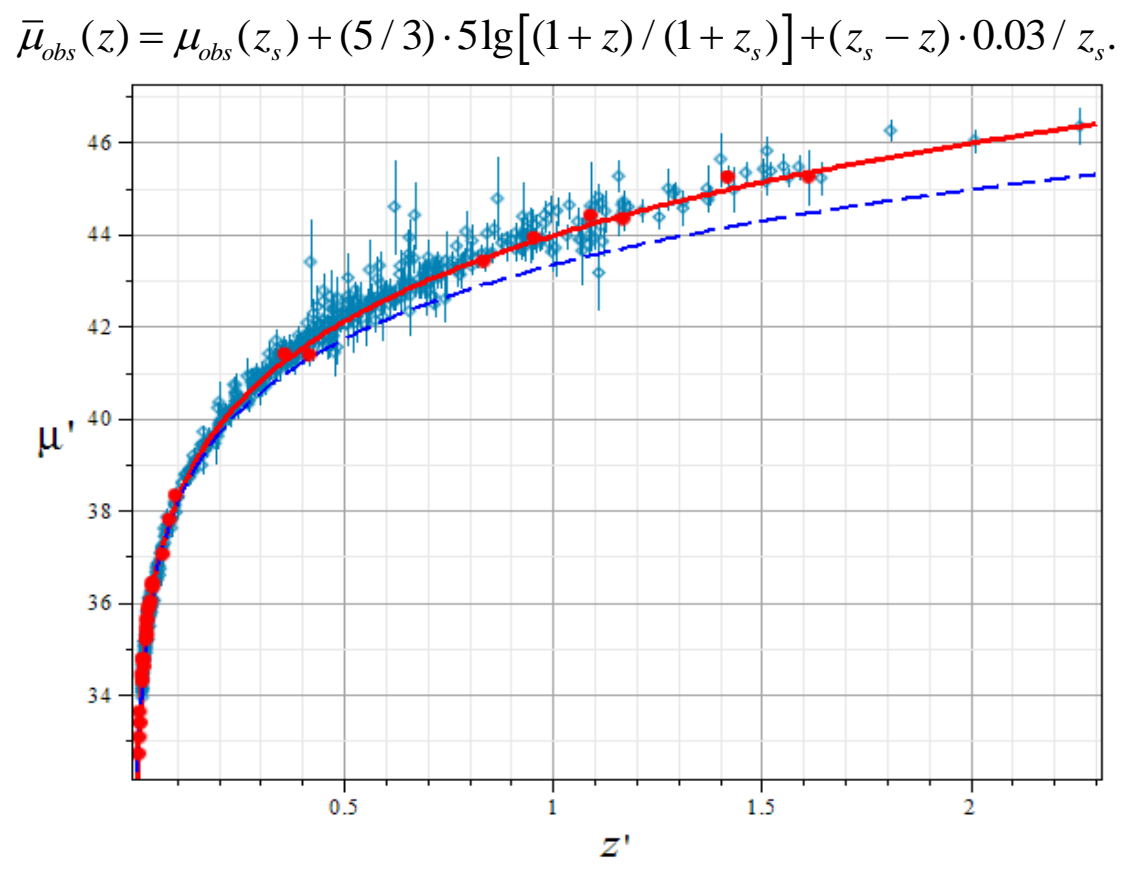

Fig. 2. The distance module - redshift diagram SNe Ia with the account of the stasis effects at $f_{0}=0.15$. Values $\bar{\mu}_{\text {obs }}(z)=\bar{m}-\bar{M}$ and $z$ are calculated by Eqs. (52)(47) for 561 SN from [10-13] (dark blue rhombs) and 33 "pure" SN from [14] (red circles). Red line - according to Eq. (16) at $h_{0}=0.734, b=0.15 \pm 0.05$. 
Comparison of the distance modulus of the new model (16) with the observations $\bar{\mu}_{o b s}(z)$ containing by the stasic effects corrections according (52) at $f_{0}=0.15$, is shown in Fig. 2 (with $\chi^{2} / N=1.16$ ).

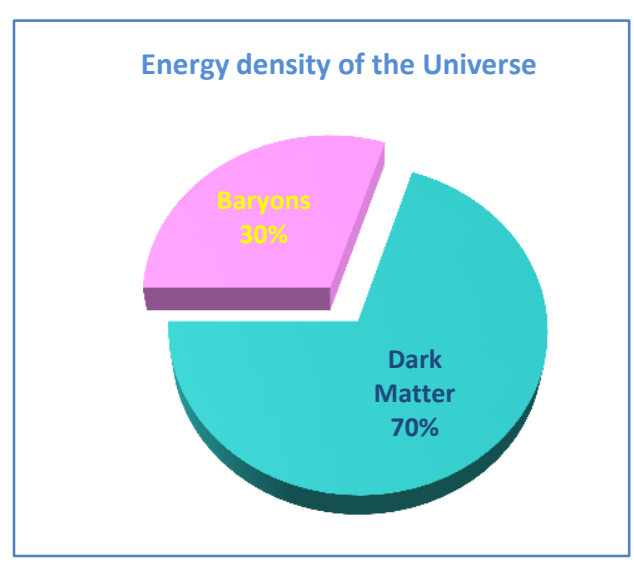

Fig. 3. Energy content of the Universe.
The parameter $h_{0}=0.734$ is fixed again in the interval $0.03<z<0.1$ with $103 \mathrm{SN} 1 \mathrm{a}$ $\left(\chi^{2} / N=0.98\right)$. At $0.20>b>0.10$ Eq. (24) gives $12.7<\tau_{0}<13.0 \mathrm{Gyr}$ for the dust period. As shows Fig. 2, at the values of parameters:

$$
h_{0}=0.734, \quad b=0.15 \pm 0.05 \text {, }
$$

the predictions of Eq. (16) at $z>0.1$ coincide with the data for $\mathrm{SN} 1$ a up to $z_{s} \sim 1.9$, $z \sim 2.5$ with $\chi^{2} / N \simeq 1.03$.

Corresponding parameters of the Universe are presented in Table 2 and Fig. 3.

\begin{tabular}{|cc|c|c|c|c|c|c|c|c|c|c|c|}
\hline$h_{0}$ & $h_{0, \text { stas }}$ & $b$ & $a$ & $a_{m}$ & $\tau$ & $\tau_{m}$ & $t$ & $t_{m}$ & $\rho_{m}$ & $\rho_{b}$ & $\rho_{d m}$ & $M$ \\
\hline 0,650 & 0,722 & 0,15 & 48 & 322 & 13,1 & 506 & 50 & 645 & 11 & $32 \%$ & $68 \%$ & 21 \\
0,650 & 0,722 & 0,20 & 41 & 203 & 12,9 & 319 & 43 & 406 & 11 & $30 \%$ & $70 \%$ & 13 \\
0,650 & 0,722 & 0,25 & 35 & 141 & 12,7 & 221 & 38 & 281 & 12 & $28 \%$ & $72 \%$ & 9 \\
\hline 0,661 & 0,734 & 0,11 & 57 & 516 & 13,0 & 811 & 58 & 1033 & 11 & $32 \%$ & $68 \%$ & 33 \\
0,661 & 0,734 & 0,18 & 43 & 237 & 12,8 & 372 & 45 & 474 & 12 & $29 \%$ & $71 \%$ & 15 \\
0,661 & 0,734 & 0,25 & 35 & 138 & 12,5 & 217 & 37 & 277 & 13 & $27 \%$ & $73 \%$ & 9 \\
\hline 0,680 & 0,756 & 0,15 & 46 & 308 & 12,5 & 484 & 48 & 616 & 12 & $29 \%$ & $71 \%$ & 20 \\
0,680 & 0,756 & 0,20 & 39 & 194 & 12,4 & 305 & 41 & 388 & 13 & $27 \%$ & $73 \%$ & 12 \\
0,680 & 0,756 & 0,25 & 34 & 134 & 12,2 & 211 & 36 & 269 & 13 & $25 \%$ & $75 \%$ & 9 \\
\hline
\end{tabular}

Table 2. Parameters of the Universe in the closed model with relativistic redshift and the stasis effects corrections. Radii $a, a_{\max }$ in Gly, ages $\tau$, $\tau_{\text {max }}, t, t_{\text {max }}$ in Gy, matter density $\rho_{m}$ in $10^{-31} \mathrm{~g} \cdot \mathrm{sm}^{-3}$, total mass $M$ in $10^{56} \mathrm{~g}$.

As it has been already noted in [8], for more exact calculation of the stasis effects the corrections to data should be studied for each observing object individually, taking into account influence of each galaxy cluster crossed by photons.

\section{Conclusion}

The presented in paper new version of the model of the closed Universe, as based on standard GR without adding to it any hypothesis, is a realistic basis for relativistic cosmology. Though starting points of the model are natural and simple, the results appear as unexpectedly successful.

At first, the closed model is theoretically most preferable case as naturally coinciding by the cosmological principle in 3-space and the fact of Big Bang. 
Secondly, at accounting of the relativistic effects the model becomes not only more consecutive, simple and exactly solvable, but describes the existing observational data without dark energy.

Thirdly, the observational discovery of additional redshift is related not by acceleration and dark energy, but by a direct appearance of the relativistic dilation of proper times at cosmological expansion.

Thus, there are significant theoretical and observational bases to consider the universe as the 3 -sphere expansion of which is decelerating.

The interpretation of this phenomenon as possible first observational revealing of the extra dimension will be developed in the next paper [15].

\section{Appendix. The Friedmann model from non-relativistic dynamics of a ball}

For clearness in those aspects of the Friedmann model which in the new version of closed model are preserved or modified, its basic relations we present in the same form in which the new version is formulated.

Simplest way to obtain Friedmann's evolution equations, as it is known, is using the Milne's Newtonian model [5] in which evolution of homogeneous dust ball of radius $r$ and mass $m=\rho \cdot 4 \pi r^{3} / 3$, where $\rho$ is mean density of matter, is described at nonrelativistic velocities of expansion. For this purpose the ball should be large enough to be homogeneous and to be expanding, but also not too large since velocity of its surface w.r.t. ball's centre should remain nonrelativistic.

In this model the total energy of unit mass comoving to ball's surface reads:

$$
\frac{1}{2}\left(\frac{d r}{d \tau}\right)^{2}-\frac{G m}{r}=E, \quad E=\text { const }
$$

where $\tau$ is time, $G$ is the gravitational constant. By inserting $r(\tau)=a(\tau) \chi$ into (54) and choosing a value of $E$, we obtain the Friedmann evolution equation for the Universe:

$$
\left(\frac{d a}{d \tau}\right)^{2}=\frac{a_{m}}{a}-1, \quad a_{m} \equiv 2 G M .
$$

From (55) and the equation for trajectory of radially propagating light:

$$
d s^{2}=d \tau^{2}-a^{2}(\tau) \cdot d \chi^{2}=0
$$

follows:

$$
\chi_{z}=\int_{a_{z}}^{a_{0}} \frac{d a}{a} \cdot \frac{d \tau}{d a}=\int_{a_{z}}^{a_{0}} \frac{d a}{\sqrt{a\left(a_{m}-a\right)}}=\arcsin \left(1-\frac{2 a_{z}}{a_{m}}\right)-\arcsin \left(1-\frac{2 a_{0}}{a_{m}}\right) .
$$

By introducing $b \equiv a_{0} / a_{m}$, we rewrite (57) in the form:

$$
\sin \chi_{z}=2 \sqrt{b} \sqrt{1-b}\left[\left(1-2 b \frac{a_{z}}{a_{0}}\right)-(1-2 b) \sqrt{\frac{a_{z}}{a_{0}}} \sqrt{\frac{1-b a_{z} / a_{0}}{1-b}}\right] .
$$

In the non-relativistic dynamics the redshift $z$ of receiving wavelength $\lambda_{r}$ w.r.t. emitted $\lambda_{e}$ and its relation to the scale factor have the form:

$$
\frac{\lambda_{r}}{\lambda_{e}}=1+z, \quad \frac{\lambda_{r}}{\lambda_{e}}=\frac{a_{0}}{a_{z}} .
$$

At a special value $b=1 / 2$ the second term in (58), proportional to $(1-2 b)$, disappears and Eq. (58) extremely simplifies: 


$$
\sin \chi_{z}=1-a_{z} / a_{0}=z /(1+z),
$$

which then leads to linear increasing of $z$ with distance.

The apparent and absolute luminosities $l, L$ of sources at lack of expansion would be related to luminosity distance $d_{p}=a \sin \chi$ as $l=L / 4 \pi d_{p}^{2}$. By the apparent and absolute magnitudes $m, M$ they are related as $l=10^{-m / 2.5} \cdot 2.52 \cdot 10^{-5} \mathrm{erg} \cdot \mathrm{sm}^{-2} \mathrm{sec}^{-1}$ and $L=10^{-M / 2.5} \cdot 3.02 \cdot 10^{35} \mathrm{erg} \cdot \mathrm{sec}^{-1}$. The expansion leads to decreasing of energy and arrival frequency of photons by factor $1+z$ :

$$
l_{F}=\frac{L}{4 \pi a_{0}^{2} \sin ^{2} \chi_{z}} \cdot \frac{1}{(1+z)^{2}} .
$$

The luminosity distance $d_{p, 0}$, thus, is equal to:

$$
d_{p, 0}=a_{0} \sin \chi \cdot(1+z)=10^{-5+(m-M) / 5} M p c,
$$

from which for the distance modulus $\mu \equiv m-M=5 \lg \left(d_{p, 0}\right)+25$ follows the expression:

$$
\mu=5 \lg \left[a_{0}(1+z) \cdot \sin \chi\right]+25 .
$$

Substitution of Eq. (59) into Eq. (58) with the account of Eq. (63), gives final "luminosity-redshift" relation of the Friedmann model $(c \neq 1)$ :

$$
\begin{gathered}
\mu=5 \lg \{2(1-b)[z-(1-2 b)(\sqrt{1+z /(1-b)}-1)]\}+A, \\
A=5 \lg \left[a_{0} /\left(b^{-1}-1\right)^{1 / 2}\right]+25=5 \lg \left(c / H_{o b s}\right)+25 .
\end{gathered}
$$

This is the standard Mattig formula, which has been written earlier through the "deceleration parameter" $q_{0}=1 / 2(1-b)$. It does not coincide with observations already at $z>0.5$ (see section 3 ).

\section{References}

1. Einstein A. (1917) Sitz. Preus. Akad. Wiss. 1, 142.

2. Friedmann A. (1922) Zs. Phys. 11, 377.

3. Lemaître G. (1927) Ann. Soc. Sci. Brux., 47A, 49.

4. Hubble E. (1929) Proc. Nat. Acad. Sci. 15 (3), 168.

5. Milne E. A. (1934) Quart. J. Math. Oxf. 5, 64.

6. Riess A. G. et al. (1998) Astron. J. 116, 1009;

7. Perlmutter S. et al. (1999) Astrophys. J. 517, 565.

8. Zakir Z. (2013) Theor. Phys. Astroph.\& Cosmol. 8(1), 1; 8(1), 7.

9. Park C.-G. (2007) arXiv:0710.1700.

10. Suzuki N. et al. (2012) Astrophys. J. 746, 85.

11. Rodney, S. A., et al. (2012) Astrophys J., 746, 1, 5.

12. Rubin D. et al. (2013) Astrophys J., 763, 1, 35, 10 pp.

13. Jones D. O., et al. (2013) arXiv:1304.0768 [astro-ph.CO].

14. Pruzhinskaya M. V. et al. (2011) Astr. L. 37, 663.

15. Zakir Z. (2013) Theor. Phys. Astroph.\& Cosmol. 8(1), 37. 